\title{
Cardiac Tamponade as Initial Presentation of Angioimmunoblastic T-Cell Lymphoma
}

\author{
Xu Zhang, MD, Ji Chao Zhang, MD, Rong Xing Liu, MD, Qi Miao, MD \\ Peking Union Medical College Hospital, Cardiac Surgery Department, Peking, China
}

\section{ABSTRACT}

A 21-year-old man presented initially with impending cardiac tamponade secondary to an angioimmunoblastic T-cell lymphoma (AITL). Following unsuccessful pericardiocentesis and subxiphoid pericardiostomy, the patient's deteriorating hemodynamics prompted an urgent sternotomy. Histopathological diagnosis confirmed AITL. While chemotherapy remains the most effective treatment of AITL, lifethreatening complications of the tumor necessitates surgical intervention.

\section{INTRODUCTION}

The characteristic features of angioimmunoblastic T-cell lymphoma (AITL) at presentation are variable. However, cardiac tamponade as the primary clinical manifestation of lymphoma is extremely rare [Lafaras 2008]. In this paper, we report the occurrence of an extrapericardial AITL in a 21-year-old man. We believe this is the second reported case of AITL presenting initially with cardiac tamponade.

\section{CASE REPORT}

A previously healthy 21-year-old man presented to our hospital emergency department with complaints of increasing dyspnea for 30 days and orthopnea for 3 days. The dyspnea was associated with dry cough, poor appetite, and fatigue. The patient stated his symptoms of dry cough and fatigue with mild dyspnea began insidiously. He was treated in a local hospital for common cold with traditional Chinese medicine without relief. On examination, the patient was pale and had difficulty breathing; he remained erect and leaned forward throughout the entire physical examination. The heart rate was 112 with evidence of a paradoxical pulse and a blood pressure of 106/60 $\mathrm{mmHg}$. The jugular veins were distended to the angle of the jaw with the patient lying at a 45 degree angle above supine. Auscultation of the chest revealed muffled heart sounds with no murmur or friction rub. Laboratory results showed a normal complete blood cell count, normal hepatic/renal function, and elevated lactate dehydrogenase.

Received Fanuary 16, 2016; received in revised form October14, 2016; accepted October 17, 2016.

Correspondence: Fi Chao Zhang, MD, Peking Union Medical College Hospital, Cardiac Surgery Department, Peking, China; (e-mail: chaojizhang@163.com).
Arterial blood gas results were: $\mathrm{PH} 7.44$, PCO2 $32 \mathrm{mmHg}$, PO2 $59 \mathrm{mmHg}, \mathrm{SAO} 292 \%$, and base excess $-1.2 \mathrm{mEq} / \mathrm{L}$. He was urgently treated with oxygen by mask, furosemide, and restricted intravenous fluids. A chest $\mathrm{x}$-ray showed cardiomegaly with a significantly broadened mediastinum and bilateral plural effusions. A 2-D echocardiogram at bedside showed a moderate nonloculated pericardial effusion. There was significant respiratory variation in the mitral and tricuspid valve inflow velocities, suggesting impending cardiac tamponade. A computed tomography (CT) scan was not able to be performed due to deteriorating hemodynamics. Right thoracentesis was performed which yielded $800 \mathrm{~mL}$ of yellow pleural effusion. Subsequent vital signs revealed a heart rate of 140-160, respiratory rate of 28-35 bpm, BP 90/50, and $83 \% \mathrm{SAO} 2$ on room air.

After unsuccessful pericardiocentesis and subxiphoid pericardiostomy, the high clinical suspicion for impending cardiac tamponade and possible resuscitation and the need for a definitive diagnosis prompted urgent surgical intervention prior to the completion of the full diagnostic workup. Following a median sternotomy, $400 \mathrm{~mL}$ of yellow pericardial effusion was drained. Surgical inspection revealed that a fishlike softtissue coating measuring 1 to $6 \mathrm{~cm}$ in thickness was present extrapericardially without intrapericardial involvement. The parietal pericardium and diaphragmatic nerves appeared to be significantly adhesive to the extrapericardial mass. As a result, radical resection was not possible and the tumor associated with the pericardium was removed from the great vessels to the diaphragm vertically and the phrenic nerve to the phrenic nerve horizontally. The central venous pressure dropped from 25 to $8 \mathrm{cmHg}$ postoperatively. Laboratory findings showed that the pleural fluid was transudative and the pericardial fluid was exudative. Cytological analysis of pleural and pericardial effusions showed no malignant cells. Pathological examination confirmed an angioimmunoblastic T-cell lymphoma. The patient had an uneventful recovery and was referred to our hematological department 7 days of postoperative care. He received 6 cycles of chemotherapy. At the 6-year followup the patient was in clinical and radiological remission.

\section{DISCUSSION}

AITL is a rare subtype of lymphoma, accounting for only $1 \%-2 \%$ of non-Hodgkin lymphomas [The Non-Hodgkin's Lymphoma Classification Project 1997]. AITL is usually characterized by generalized peripheral lymphadenopathy, but many patients have presentations of extranodal involvement. The most frequently involved extranodal sites include 
the bone marrow, spleen, skin, and lungs. Among the commonly reported presenting symptoms and signs, ascites/effusion account for $26 \%$ [Mourad 2008]. In our patient, pleural and pericardial involvement were the first presenting signs. Pleural effusion is a relatively common finding in patients with AITL. However, very few cases of AITL accompanied by pericardial effusion have been reported. To our knowledge, only one case of AITL with primary cardiac tamponade has been described [Lafaras 2008]. In that case, a 59 year-old man presented with cardiac tamponade as the initial clinical manifestation of AITL along with malignant pleural mesothelioma. The patient's critical cardiac condition resolved after subxiphoid pericardiocentesis, in contrast to our patient, who underwent urgent removal of AITL and pericardiectomy via median sternotomy after unsuccessful pericardiocentesis and subxiphoid pericardiostomy.

Overall, cardiac tamponade may derive from the accumulation of any intrapericardial contents to a degree sufficient to compress the heart and interfere with cardiac filling during diastole. In the present case, the effects of the diffuse extrapericardial tumor resulting in cardiorespiratory embarrassment included displacement and severe compression of the heart and major vessels and effusions in the pericardial and pleural cavities. Although the volume of pericardial effusion in our patient was relatively minimal, the symptoms were severe because the extrapericardial tumor coating markedly impeded compensated expansion of the pericardium.

The diagnosis of AITL is established on the basis of histologic evaluation of biopsy specimens. These can be obtained via image-guided or open biopsies. The most diagnostic methods for stable patients include various imaging modalities and pathohistology. Ultrasonic Cardiography (UCG) can asses heart function and intrapericardial effusion as well as detect tumors adjacent to the cardiac valves and main vessels, but can neglect to evaluate extrapericardial tissues. MRI or CT assures superior imaging of potential pericardial involvement. Pathohistological diagnosis along with MRI and CT results are used for tumor staging, which allow for more specific and less toxic therapies [Ceresoli 1997].

\section{CONCLUSION}

Chemotherapy is the mainstay of treatment for lymphoma. In our patient, surgical treatment provided a window of opportunity in which chemotherapy could take effect. We thought that the threatening cardiac tamponade was grounds for urgent drainage of pericardial effusion and removal of the AITL. Surgical intervention should remain an option for patients with severe symptomatic pericardial effusion associated with this type of tumor.

\section{REFERENCES}

Lafaras C, Mandala E, Venizelos I, et al. 2008. Cardiac tamponade as primary manifestation of angioimmunoblastic T-cell lymphoma (AITL). Coexistence with malignant mesothelioma. Onkologie 31:546-548.

The Non-Hodgkin's Lymphoma Classification Project. 1997. A clinical evaluation of the International Lymphoma Study Group classification of non-Hodgkin's lymphoma. Blood 89: 3909-18.

Mourad N, Mounier N, Briere J, et al. 2008. Clinical, biologic, and pathologic features in 157 patients with angioimmunoblastic T-cell lymphoma treated within the Groupe d'Etude des Lymphomes de l'Adulte (GELA) trials. Blood 111:4463-4470.

Ceresoli GL, Ferreri AJ, Bucci E, et al. 1997. Primary cardiac lymphoma in immunocompetent patients: diagnostic and therapeutic management. Cancer 80:1497-506. 